\section{LASERS}

\section{Heating Deuterium}

ONE attractive proposal for a thermonuclear reactor that has emerged during the past few years has involved the heating of a solid pellet of deuterium, or deuterium and tritium, with a laser beam, so that a thermonuclear reaction occurs during the very rapid expansion phase. Apart from the intriguing problem of how to operate a trapdoor arrangement at such a speed that the laser is not demolished by the thermonuclear shock-wave, one serious drawback of the idea is that the pellet might not absorb energy and that available lasers might not be sufficiently energetic (see J. L. Tuck, Nature, 233, 593 ; 1971).

One of the stumbling blocks has been that a deuterium-tritium pellet at $4 \mathrm{~K}$, for example, has a density of $6 \times 10^{22}$ atoms $\mathrm{cm}^{-3}$, which suggests that the plasma frequency would be so high that no laser radiation could be absorbed. But in a recent issue of Physical Review Letters $(\mathbf{2 8}, 85 ; \mathbf{1 9 7 2})$, R. P. Godwin of the Los Alamos Scientific Laboratory presents a model to explain recent experimental indications that total reflexion is not always the inevitable outcome of shining a laser beam onto a deuterium pellet.

The stimulus for Godwin's work came from a "workshop" on laser interactions which was held last September at the Rensselaer Polytechnic Institute (the proceedings of which are to be published). At that gathering, $F$. Floux reported that he had observed energy absorption of 64 per cent when a $44 \mathrm{~J}$ Nd-glass laser pulse was fired at a deuterium pellet; this absorption was accompanied by the production of neutrons, X-rays and energetic ions. Other work reported at the conference last September also suggested that it was necessary to administer a small amount of energy to the laser target just before the laser pulse proper (in the form of a prepulse or of a shaped leading edge for the main pulse).

Godwin has suggested that the absorption can be understood in terms of a model in which the plasma sample has on its surface a small amount of plasma with a low density and a plasma frequency equal to the laser frequency; the remainder of the sample, he postulates, has the high density and high plasma frequency expected for a deuterium pellet. Godwin first performs some calculations on a system beloved of the teachers of undergraduate courses in electromagnetic theory-a thin film with polarized radiation impinging upon it at an angle. The assumptions made in this case are that the thin film is a plasma, the density of which is such that the frequency of the electromagnetic radiation is equal to the plasma frequency, and that the complex dielectric constant is that of a slightly damped Drude free electron gas. It turns out that the maximum absorption under these circumstances is 50 per cent and occurs when the thickness of the film is half of the wavelength of the electromagnetic (laser) radiation.

If such a film is now imagined to be backed by plasma of higher density, which acts like an almost perfect conductor and therefore like a mirror, the electrostatic method of images leads one to the conclusion that maximum absorption ( 100 per cent this time) should occur when the film is a quarter of a wavelength thick. (For unpolarized laser light, of course, the maximum absorption is only 50 per cent.)

Godwin presents this "hot spot" model as the basis for an understanding of how Floux has managed to achieve absorption of laser radiation in a solid deuterium sample. Plainly, the use of a precursor pulse is the crux of the matter, for it provides the energy both to ionize atoms on and near the surface and to increase their average separations so that the electron density is $\sim 10^{21} \mathrm{~cm}^{-3}$, the critical value for which the plasma frequency is equal to the frequency of the light from an $\mathrm{Nd}$ glass laser.

As far as the maintenance of a thermonuclear reaction is concerned, of course, it is the energy available to the deuterium (or deuterium and tritium) ions that is important. Under ideal conditions and with a laser pulse energy of $10 \mathrm{~J}$, Godwin calculates that the energy available for each particle is about $10 \mathrm{MeV}$. But lest anybody should think that controlled thermonuclear fusion on a practical scale is just around the corner, he hurriedly qualifies this by saying that when energy loss mechanisms such as thermal conduction are taken into account the particle energies will certainly not be in the $\mathrm{MeV}$ region. He does not, however, make an estimate of what the particle energies will be. In his article in Nature, Tuck mentioned threshold temperatures of about $10 \mathrm{keV}$ for deuterium-tritium thermo. nuclear reactions and about $50 \mathrm{keV}$ for those between deuterium and deuterium.

\section{PESTICIDES \\ Mosquito Control}

from a Correspondent

NEARLy all methods of mosquito control in operation at present are based on the use of insecticides. In spite of the growing problem of resistance to chlorinated hydrocarbons, organophosphates and to carbamates the campaigns against mosquito-borne diseases still depend on the use of residual insecticides generally and DDT in particular. Sanitary measures of reduction of breeding sites are not economically feasible in rural areas of developing countries and biological control offers some promise but little immediate help. Thus any withdrawal of insecticides from public health use would jeopardize nearly all public health programmes aiming at the eradication or control of malaria, yellow fever, dengue, virus encephalitis and filariasis-all mosquito-borne diseases. These were the principal points of a symposium on problems of mosquito control convened in London on January

\title{
Two-headed Enzyme from Horse Muscle
}

As part of a long-term programme of structure determination of all the enzymes in a metabolic pathway, Blake, Evans and Scopes have studied the glycolytic enzyme, phosphoglycerate kinase, from horse muscle, and its structural features at $6 \AA$ resolution are reported in next Wednesday's Nature New Biology (February 16). The protein is a single polypeptide chain with the rather unusually high molecular weight of 48,000 . The electron density maps show that it is in effect made up of two globular subunits, joined together by a narrow neck. The globular portions correspond in size to a molecular weight of some 20,000 each, one being elongated, the other nearly spherical. This leaves 8,000 for the neck, which is about $20 \AA$ thick, and in which helixlike structure can be made out.

In an attempt to locate the active centre, magnesium and ATP, one of the substrates, were allowed to diffuse into the crystals. This, however, proved unsuccessful, for the crystal cracked, indicating that a conformational change in the enzyme had occurred. With magnesium and ADP, on the other hand, isomorphous patterns were obtained, and the electron density difference map showed only a single area of positive intensity, corresponding to the magnesium-ADP.

The profile has two lobes, which are surmised to represent the nucleoside and the magnesium-diphosphate parts of the magnesium-ADP complex. One of the lobes dips into a shallow depression in the protein, and the other into the solvent. The binding site is in the more elongated of the two globules and so far no function for the other subunit has been found. The speculation is that it has either a modifying function on the activity, or that it is implicated in an interaction with some other enzyme in the pathway. 